\title{
Síndrome de Cotard en una paciente con lupus eritematoso sistémico y meningioma petroclival izquierdo
}

\author{
Giovanni Pitta $^{1}$ (D), Marcelo O'Higgins ${ }^{2}$ iD , Noelia Ruiz Díaz ${ }^{2}$ iD, Johana Silvero ${ }^{1}$, \\ José Almirón-Santacruz ${ }^{\text {iD }}$, Santiago Báez ${ }^{1}$, Miguel Vera1 ${ }^{1 D}$, *Julio Torales ${ }^{2,3}$ (D) \\ ${ }^{1}$ Universidad Nacional de Asunción, Facultad de Ciencias Médicas, I Cátedra de Clínica Médica. \\ San Lorenzo, Paraguay \\ ²Universidad Nacional de Asunción, Facultad de Ciencias Médicas, Cátedra de Psiquiatría. San \\ Lorenzo, Paraguay \\ ${ }^{3}$ Universidad Nacional de Asunción, Facultad de Ciencias Médicas, Cátedra de Socioantropología \\ (Filial de Santa Rosa). Santa Rosa del Aguaray, Paraguay
}

Cómo referenciar este artículo/ How to reference this article:
Pitta G, O'Higgins M, Ruiz Díaz N, Silvero J, Almirón-Santacruz J, Báez S, Vera M, Torales J. Síndrome de Cotard en una paciente con lupus eritematoso sistémico y meningioma petroclival izquierdo. Mem. Inst. Investig. Cienc. Salud. $2021 ; 19(1)$ : 74-78

\section{RE S U M E N}

El síndrome de Cotard es una entidad rara en la cual el paciente manifiesta ideas delirantes acerca de que se encuentra sin vida o que sus órganos internos se encuentran en estado de descomposición, entre otras alteraciones psicopatológicas. Existen diferentes reportes sobre el síndrome en diversas culturas y poblaciones. Usualmente tiene presentaciones neuropsiquiátricas y se lo identifica acompañando entidades neurológicas, metabólicas, infecciosas, entre otras. A través de este reporte de caso, se presenta una paciente con lupus eritematoso sistémico, que desarrolló este tipo de ideas delirantes y durante su hospitalización se detectó un meningioma petroclival izquierdo. Finalmente, se llegó a la conclusión de que no se encontraba en actividad lúpica y que la ubicación del tumor no sería la causante de la alteración conductual. El Síndrome de Cotard es un trastorno neuropsiquiátrico poco común, que debe ser considerado ante la existencia de ideas delirantes de tipo nihilistas.

Palabras clave: Lupus Eritematoso Sistémico, meningioma, delusiones.

\section{Cotard's syndrome in a female patient with systemic lupus erythematosus and left petroclival meningioma}

\section{ABSTRACT}

Cotard's syndrome is a rare entity in which the patient manifests delusional ideas about being lifeless or that his/her internal organs are in a state of decomposition, among other psychopathological alterations. There are different reports in different cultures and populations about the syndrome. It usually has neuropsychiatric presentations and is identified by accompanying neurological, metabolic, and infectious entities, among others. In this case report, a female patient with systemic lupus erythematosus is presented, who developed this type of delusions and during her hospitalization a left petroclival meningioma was detected. Finally, it was concluded that she was not in lupus activity and that the location of the tumor was not the cause of the behavioral alteration. Cotard's syndrome is a rare neuropsychiatric disorder, which should be considered when there are nihilistic delusions.

Keywords: Systemic Lupus Erythematosus, meningioma, delusions. 


\section{INTRODUCCIÓN}

El síndrome de Cotard es una entidad conocida por parte de la psiquiatría. La entidad fue descrita primeramente en el año 1880, por parte de Jules Cotard, en una mujer de 43 años que presentó una "melancolía ansiosa" con ideas delirantes de tipo "hipocondríacas"(1).

Sobre la prevalencia del síndrome de Cotard y su epidemiología, resulta difícil de determinar debido a que la mayoría de los reportes consisten en series de casos o reportes de casos individuales ${ }^{(2)}$, pero considerando una muestra en México en el Instituto Nacional de Neurología y Neurocirugía se encontró una prevalencia del $0,11 \%$ en pacientes neurológicos y de $0,62 \%$ en pacientes psiquiátricos de dicho instituto ${ }^{(3)}$.

Desde entonces se ha asociado a múltiples afecciones médicas con componentes neuropsiquiátricos. Si bien su presentación puede ser descrita como la presencia de ideas delirantes de tipo nihilista o de negación, en las cuales el paciente niega su propia existencia o que se mantenga con vida ${ }^{(4)}$, su presentación puede variar dependiendo del cuadro con el que se acompaña. Se considera una entidad rara y sigue siendo poco comprendida ${ }^{(1)}$.

Este caso consiste en el de una paciente de sexo femenino de sesenta años de edad que presenta ideas delirantes nihilistas hasta el extremo de negarse a alimentarse y de presentar cuadros de gran agitación psicomotriz al tratar de asistirla. Esta paciente presenta una clínica de diez años de evolución del diagnóstico de lupus eritematoso sistémico y durante la internación se descubre un meningioma petroclival izquierdo.

\section{Presentación del caso}

Paciente de sexo femenino de sesenta años de edad con diagnóstico de base lupus eritematoso sistémico desde hace 10 años con abandono de tratamiento un mes antes del ingreso y trastorno depresivo con seguimiento irregular. Acude a urgencia por cuadro de una semana de evolución de agitación psicomotriz junto con lenguaje incoherente, negación a recibir medicación, agresividad e ideas delirantes de tipo nihilista.

Al examen físico no presenta datos positivos de valor y al laboratorio presenta hemograma, perfil renal, electrolitos, perfil hepático y orina simple en rango. Dado el cuadro de base, se sospecha de una psicosis lúpica por lo que se solicitan marcadores de actividad que retornan en rango (Tabla 1 ).

Tabla 1: Serología reumatológica e infecciosa de la paciente

\begin{tabular}{ccc}
\hline Serología & Resultado & Rango \\
\hline ANA & $1: 80$ & $1: 80$ \\
Anti DNA & $1: 10$ & $1: 10$ \\
C3 & 97 & $88-201 \mathrm{mg} / \mathrm{DI}$ \\
C4 & 32 & $15-45 \mathrm{mg} / \mathrm{dL}$ \\
PCR & 1 & $<6 \mathrm{mg} / \mathrm{dL}$ \\
VIH & No reactiva & No reactiva \\
VDRL & Reactiva 18 & No reactiva \\
FTA abs IgM & Negativo & Negativo \\
FTA abs IgG & Negativo & Negativo \\
HVBsAg & 0,1 & $<1$ \\
Anti HVC & 0 & $<1$ \\
\hline
\end{tabular}

Se realiza una punción lumbar que presenta un citoquímico normal y cultivo negativo a las 48 horas. Además de serologías virales no reactivas.

Se realiza una resonancia magnética contrastada de encéfalo que informa signos de meningioma petroclival izquierdo con invasión de seno cavernoso ipsilateral, sin edema perilesional, sin compresión ventricular ni alteración del resto de las regiones cerebrales. En búsqueda de foco epileptógeno secundario a tumor se realiza un electroencefalograma y se inicia tratamiento con ácido valproico a $1500 \mathrm{mg}$ al día. Es evaluada por neurocirugía que propone como causa de la alteración psiquiátrica un trastorno independiente al proceso tumoral dado la ubicación de este y su poca 
correlación con la clínica. Debido a lo anterior, se decide que la paciente sea evaluada por el servicio de psiquiatría.

En la evaluación psiquiátrica, los familiares de la paciente refieren que el cuadro se inicia aproximadamente un mes antes del ingreso, cuando la paciente comunica a sus familiares que desea suspender su tratamiento farmacológico de lupus eritematoso sistémico. Tras esto el cuadro fue avanzando progresivamente con disminución de la ingesta de alimentos, refería molestias gastrointestinales, indicaba a sus familiares que su "estomago se achicaba". Se le realizan diversas evaluaciones clínicas por parte de facultativos que le indican que no encuentran alteraciones; sin embargo, la paciente refería que ya no sentía sus órganos internos, que era un zombie y que se encontraba muerta. Los familiares llevan a la paciente junto a su madre al interior del país. En este lugar, la paciente presenta episodios de agitación psicomotriz, se rehúsa a dormir, comer o vestirse puesto que manifiesta encontrarse fallecida y que todas estas acciones carecen de sentido. Los familiares la traen a la Urgencia y es ingresada en el Servicio de Medicina Interna.

Durante la internación, la paciente sigue indicando a sus familiares que se encuentra "muerta", por momentos no reconoce a los mismos, no acepta que tiene hijos puesto que se encuentra fallecida, indica que la sangre no circula por su cuerpo y que sus órganos internos se encuentran en descomposición. La paciente oscila entre estados que van desde agitación psicomotriz intensa (se trata de remover las sondas o se muestra heteroagresiva con objetos) hasta estado de negativismo activo y mutismo.

La evaluación de la paciente se realizó en diferentes espacios, siendo la primera evaluación en el Departamento de Emergencias del Hospital de Clínicas en donde se le indica haloperidol $10 \mathrm{mg} /$ día vía oral y en caso de agitación psicomotriz haloperidol $5 \mathrm{mg}$ y midazolam $5 \mathrm{mg}$ en forma intramuscular.

En la primera evaluación, el examen psíquico constata que la paciente se encuentra vigil, ubicada en tiempo, parcialmente en espacio y persona, atención hipoproséxica, lenguaje de tono bajo, bien articulado, humor hipotímico, afecto aplanado. Pensamiento perseverante, con ideas delirantes de tipo nihilista: "estoy muerta, no como porque estoy muerta". No se encuentran alteraciones sensoperceptivas al momento del examen e introspección nula.

La paciente es estabilizada clínicamente en sala de Medicina Interna por 9 días, siendo luego trasladada al Servicio de Psiquiatría, donde permanece internada por 30 días. La impresión diagnóstica al ingreso fue de un síndrome de Cotard, en el contexto de un trastorno depresivo mayor con síntomas psicóticos; sin embargo, durante la internación, la paciente desarrolló un episodio maníaco, por lo que se decide ajustar el esquema de medicación, con lo que el cuadro termina cediendo. Al alta, la impresión diagnóstica fue de un síndrome de Cotard, en el contexto de un trastorno bipolar tipo I. La medicación al alta quedó establecida en olanzapina $5 \mathrm{mg} /$ día, valproato $750 \mathrm{mg} /$ día y quetiapina $50 \mathrm{mg} /$ día. Se agendó a la paciente para seguimiento por consultorio externo, con buena evolución.

\section{DISCUSIÓN}

El síndrome de Cotard desde su primera descripción se ha asociado a múltiples entidades psiquiátricas como también de otras áreas de la medicina. Se han descrito cuadros dentro de depresión severa, hipocondría y psicosis extremas ${ }^{(1)}$, esquizofrenia ${ }^{(2)}$, demencias ${ }^{(5,6)}$, delirium hipoactivo ${ }^{(7)}$ como fue también descrito en la encefalitis anti-NMDA ${ }^{(8)}$, epilepsia ${ }^{(9)}$, tumores del sistema nervioso central ${ }^{(10)}$, encefalopatía de Hashimoto ${ }^{(11)}$ y la enfermedad de Parkinson ${ }^{(12)}$.

En el caso de esta paciente se asocia el diagnóstico de lupus eritematoso sistémico desde hace diez años que no corresponde a actividad con toque neuropsiquiátrico. Si bien puede ser la psicosis la única manifestación, no es la alteración más frecuente y ésta generalmente se expresa junto con una serología que constate la actividad lúpica; que en el caso de la paciente se encontraba negativa ${ }^{(13)}$.

Además del LES, se tiene el hallazgo en la internación actual de un meningioma petroclival. Se ha observado la presencia de síntomas compatibles con el síndrome de 
Cotard en pacientes con tumores del lóbulo parietal o en casos de epilepsia secundaria a tumores ${ }^{(10)}$. Si bien en este caso el Departamento de Neurocirugía descartó que la alteración conductual se deba a la presencia del tumor, se debe tener en cuenta que esto pudo ser un factor más que predispuso al desarrollo del cuadro.

En cuanto al tratamiento planteado, la utilización de haloperidol como tratamiento en este caso se fundamentó más que nada en el tratamiento de los cuadros de agitación psicomotriz. Si bien la literatura menciona la utilización de olanzapina, aripiprazol, clozapina y sulpiride con buena respuesta en monoterapia ${ }^{(4)}$, se menciona igualmente a la combinación de haloperidol con mirtazapina y clomipramina ${ }^{(14)}$, estos últimos en el caso de cuadros depresivos intensos con síntomas psicóticos englobados en un Síndrome de Cotard. De igual manera se plantea una buena respuesta al tratamiento con inhibidores selectivos de la recaptación de serotonina en monoterapia(15). Sin embargo, se describe la mejor respuesta con la aplicación de terapia electroconvulsiva ${ }^{(16)}$.

Considerando estos datos, tener al síndrome de Cotard como una posible manifestación neuropsiquiátrica de entidades diversas dentro de la medicina permite tener una mirada más completa a la presencia de alteraciones conductuales en pacientes con múltiples comorbilidades. Existen diferentes alteraciones del comportamiento, de la cognición y de la expresión de la personalidad que tienen como base problemas complejos que involucran afecciones sistémicas y conviene tenerlas en cuenta al abordar este tipo de pacientes.

\section{REFERENCIAS BIBLIOGRÁFICAS}

1. Dieguez S. Cotard Syndrome. Front Neurol Neurosci. 2018; 42: 23-34. $10.1159 / 000475679$

2. Huarcaya-Victoria J, LedesmaGastañadui M, Huete-Cordova M. Cotard's Syndrome in a Patient with Schizophrenia: Case Report and Review of the Literature. Case Rep Psychiatry. 2016; 2016: 6968409. 10.1155/2016/6968409

3. Ramirez-Bermudez J, Aguilar-Venegas LC, Crail-Melendez D, Espinola-Nadurille M, Nente F, Mendez MF. Cotard syndrome in neurological and psychiatric patients. J Neuropsychiatry Clin Neurosci. 2010; 22(4):409-416. 10.1176/jnp.2010.22.4.409

4. Tomasetti $C$, Valchera $A$, Fornaro $M$, Vellante F, Orsolini L, Carano A, et al. The 'dead man walking' disorder: an update on Cotard's syndrome. Int Rev Psychiatry. 2020; 32 (5-6): 500-509. 10.1080/09540261.2020.1769881

5. Cipriani G, Nuti A, Danti S, Picchi L, Di Fiorino M. 'I am dead': Cotard syndrome and dementia. Int J Psychiatry Clin Pract. 2019;23(2):149156. 10.1080/13651501.2018.1529248

6. Pereira CW, Florentino ISM, Machado L. When Dementia Kills Before One Passes Away: Case Report of Cotard's Syndrome. Clin Schizophr Relat Psychoses. 2018. Epub ahead of print. https: $10.3371 /$ CSRP.PEFL.061518

7. Oberndorfer R, Schönauer C, Eichbauer $H$, Klaushofer K, Friedrich F. Cotard Syndrome in Hypoactive Delirium - A Case Report. Psychiatr Danub. 2017;

29(4):500-502.

10.24869/psyd.2017.500

8. Funayama $M$, Takata $T$, Mimura $M$. Cotard's syndrome in anti-N-methyl-daspartate receptor encephalitis. Psychiatry Clin Neurosci. 2018; 72(6):455-456. 10.1111/pcn.12658

9. Gil YE, Choi JY, Kim TJ, Park SA, Huh K. Post-ictal Cotard delusion in focal epilepsy patients. Seizure. 2019; 71:80-82.

10.1016/j.seizure.2019.06.015

10. Bhatia MS. Cotard's Syndrome in parietal lobe tumor. Indian Pediatr. 1993; 30(8):1019-1021.

11. Hajnal L, Lazary J. Hashimotoenkefalopátiához társuló Cotardszindróma: esettanulmány [Cotardsyndrome associated with Hashimoto encephalopathy: a case report]. Neuropsychopharmacol Hung. 2019; 21(2):85-93.

12. Solla P, Cannas A, Orofino G, Marrosu F. Fluctuating Cotard syndrome in a patient with advanced Parkinson disease. Neurologist. 2015; 19(3):7072. 10.1097/NRL.0000000000000010

13. Zhang $\mathrm{Y}$, Han $\mathrm{H}$, Chu L. Neuropsychiatric Lupus Erythematosus: Future Directions and Challenges; a Systematic Review and Survey. Clinics 2020; 75: e1515. 10.6061/clinics/2020/e1515

14. Sahoo A, Josephs KA. A Neuropsychiatric Analysis of the Cotard Delusion. J Neuropsychiatry Clin Neurosci. 2018; 30(1):58-65. 10.1176/appi.neuropsych. 17010018 
15. Moschopoulos NP, Kaprinis S, Nimatoudis J. Cotard's syndrome: Case report and a brief review of literature. Psychiatriki. 2016; 27(4):296-302.

10.22365/jpsych.2016.274.296
16. Mamaeva T, Christensen DS, Nielsen CT. Electroconvulsive therapy is efficient in treating Cotard's syndrome. Ugeskr Laeger. 2016; 178(51): V04160230. 